\title{
//Resumen:
}

Actualmente en Colombia los programas de vivienda de interés social se han convertido en uno de los programas bandera de los planes de desarrollo desde el nivel nacional hasta el local. Con relación a los aspectos de vivienda de interés social, y más desde el punto de vista sustentable vale la pena destacar que los gobiernos han tratado de menguar o fortalecer este aspecto de alguna $u$ otra forma a través de proyectos y planes de desarrollos más positivo orientados a una población que cada vez se encuentra en una situación de vulnerabilidad menos sostenible.

El presente articulo pretende enfatizar para un mayor entendimiento y aceptación de la comunidad sobre el tema de la vivienda, asignándole una valoración y definición de una realidad social que se vive actualmente con relación a la vivienda de interés social. El hombre en general, y, en especial la población que se encuentra ubicada en el Distrito de Barranquilla Atlántico, es un ser ávido de anhelos y esperanzas por alcanzar un mundo pletórico de bienestar para su familia, lo que se hace evidente en el desarrollo de su diario vivir, cargado de un sinnúmero de esfuerzos, de sacrificios que lo llevan dentro del marco de sus posibilidades a llenarse de imaginación, creatividad, astucia, y en ocasiones en el campo de la temeridad a correr riesgos que le colocan ante el peligro de perder su dignidad, su libertad y hasta la forma de su propio hábitat el cual es un estado de vulnerabilidad alcanzado hoy en día en Colombia.

\section{Análisis y caracterización de la vivienda de interés social mínima sustentable para la ciudad de Barranquilla - Colombia [Carlos Augusto Rengifo Espinosa]}

\section{//Fecha de recepción [30 de mayo de 2011] //Fecha de aceptación [1 septiembre de 2011]}

\section{//Palabras clave:}

Programas habitacionales, Vivienda de Interés social, Espacios habitacionales, Ley urbana, Gobierno de la ciudad.

\section{//Keywords:}

Habitationals programs, Social Housing, Space habitational, Law urban, Government of the city. 
Al desarrollar estos "Planes de Vivienda" que se ha denominado "Vivienda de Interés Social", el gobierno y los constructores han tomado como parámetros de ejecución, el que sea una vivienda barata, de bajo costo por unidad de metro cuadrado en cuanto al valor del terreno y construcción utilizando de manera general materiales cuyo reducido valor hace que finalmente ésta característica de vivienda barata esté representada en una baja calidad de obra, durabilidad y poco amigable con el medio ambiente, sin contar con los efectos que sobre la salud mental y el bienestar de sus moradores pueda ocasionar por la limitación del espacio, la libertad e intimidad; ya que el sólo hecho de enfocar la construcción de este tipo de vivienda bajo estos parámetros de economía y de reducción al máximo de espacio por unidad de vivienda familiar, ya que el área promedio es de $6.00 \mathrm{Mts}$, ancho por $12.00 \mathrm{Mts}$, de largo, conllevará de por sí un factor grave en deterioro de la comodidad habitacional para las personas que la habitarán luego, creándoles un ambiente muy estrecho para su desempeño, formando un cierto hacinamiento promiscuo para el normal desarrollo de la familia.

Con el propósito de conocer y analizar las características de la vivienda sustentable de interés social en la ciudad de Barranquilla, el presente trabajo criterios arquitectónicos bioclimáticos y ecológicos a utilizar en una vivienda, se acogerá la premisa "Ciclo de vida de los materiales: Materiales con bajo contenido energético, bajas emisiones de gases y el mayor porcentaje de materia reciclables" como la de mayor importancia y se desarrolla una propuesta de solución a la problemática de hacinamiento, saludable y sustentable para la ciudad de Barranquilla, por lo que se requiere establecer líneas de investigación y práctica profesionales prioritariamente en los programas masivos Vivienda de Interés Social.

\section{//Abstract:}

In China and in ancient Egypt were rudimentary machines connected by a bar hoops and some bamboo rolling, but the story goes that close to 1490 could be seen some ketches of a bicycle with a chain drive pedal-powered, then how is it possible for more than 500 years later, bicycle paths have been outlined in $97 \%$ of our streets and racing?

Currently in Colombia, programs of social interest housing have become one of the programs flag of development plans from the national level to the local. In relation to aspects of social housing, and more from the point of view sustainable it is worth noting that governments have sought to undermine or strengthen this aspect in some form or other through projects and plans for developments more positive oriented to a population that is increasingly being found in a situation of vulnerability less sustainable.

This article aims to highlight for a greater understanding and acceptance of the community on the topic of housing, by assigning a valuation and definition of a social reality that is now live in relation to the social housing. Man in general, and, in particular the people that is located in the district of Barranquilla Atlantic, is a be avid longings and hopes to achieve a world bursting with welfare for your family, which is evident in the development of their daily lives, filled with a myriad of efforts, sacrifices to carry within the framework of its possibilities to be filled with imagination, creativity, cunning, and sometimes in the field of the temerity to take on the risks that you placed In the face of danger of losing their dignity, freedom and even the way of their own habitat which is a state of vulnerability reached today in Colombia.

In developing these "housing plans" that has been called "social interest housing", the government and the manufacturers have taken as execution parameters, which is a low-cost housing, low-cost per unit of square meter in regard to the value of the land and construction using in a general way materials whose reduced value eventually causes this feature of housing It gets this represented at a low quality of work, durability and not very user-friendly to the environment, without the effects on mental health and well-being of its inhabitants might be caused by the limitation of space, freedom and privacy; since the mere fact of focusing the construction of this type of housing under these parameters of the economy and reduction to the maximum amount of space per unit of family housing, since the average area is $6.00 \mathrm{Mts}$, width by $12.00 \mathrm{Mts}$, long, Will entail in itself a major factor in deterioration of the comfort housing for the people who will inhabit then, creating an environment very close to its performance, forming a true overcrowding promiscuous for the normal development of the family.

With the purpose of knowing and analyze the characteristics of the sustainable housing of social interest in the city of Barranquilla, the present work architectural criteria bioclimatic and ecological to use in a housing, it will host the premise "life-cycle of materials: materials with low energy content, low-emission of gases and the highest percentage of recyclable material" to be the most important and develops a proposal for a solution to the problem of overcrowding, Healthy and sustainable for the city of Barranquilla, so we need to establish lines of research and professional practice primarily in massive programs of social housing. 
ARTE \& DISEÑO, ISSN: 1692-8555, Vol. 9 Núm. 2, Julio - Diciembre de 2011 Análisis y caracterización de la vivienda de interés social mínima sustentable para la ciudad de Barranquilla - Colombia, págs. 38-48

\section{INTRUDUCCIÓN}

En Colombia los programas de vivienda de interés social se han convertido en uno de los programas bandera de los planes de desarrollo desde el nivel nacional hasta el local. Con relación a los aspectos de vivienda de interés social, y más desde el punto de vista sustentable vale la pena destacar que los gobiernos han tratado de menguar o fortalecer este aspecto de alguna $u$ otra forma a través de proyectos y planes de desarrollos más positivo orientados a una población que cada vez se encuentra en una situación de vulnerabilidad menos sostenible. Al desarrollar estos "Planes de Vivienda" que se ha denominado "Vivienda de Interés Social", el gobierno y los constructores han tomado como parámetros de ejecución, el que sea una vivienda barata, de bajo costo por unidad de metro cuadrado en cuanto al valor del terreno y construcción utilizando de manera general materiales cuyo reducido valor hace que finalmente ésta característica de vivienda barata esté representada en una baja calidad de obra, durabilidad y poco amigable con el medio ambiente, sin contar con los efectos que sobre la salud mental y el bienestar de sus moradores pueda ocasionar por la limitación del espacio, la libertad e intimidad; ya que el sólo hecho de enfocar la construcción de este tipo de vivienda bajo estos parámetros de economía y de reducción al máximo de espacio por unidad de vivienda familiar.

Con el propósito de conocer y analizar las características de la vivienda sustentable de interés social en la ciudad de Barranquilla, el presente trabajo, recoge además del planteamiento del problema, el marco teórico y legal como referente para la investigación. La metodología y criterios arquitectónicos bioclimáticos y ecológicos a utilizar en una vivienda, se acogerá la premisa "Ciclo de vida de los materiales: Materiales con bajo contenido energético, bajas emisiones de gases y el mayor porcentaje de materia reciclables" como la de mayor importancia y se desarrolla una propuesta de solución a la problemática de hacinamiento, saludable ${ }^{1}$ y sustentable para la ciudad de Barranquilla.

\section{MARCO TEÓRICO}

Vivienda y Calidad de Vida. En la comprensión de la dinámica del uso del espacio doméstico Teresa 0ntineros como se ha tenido la tendencia a considerar la casa, es decir el espacio doméstico en un sentido arquitectónico y antropológico como el espacio físico que tiene la condición de albergue, dejando de lado las significaciones y las expresiones de quienes la construye.

La arquitectura en la Humanización de la familia a través de la vivienda. Roizbblatt, Arturo et al, llegaron a la conclusión que se requieren más estudios que permitan en función de las características culturales, sociodemográficas y económicas de la región comprender el impacto de la vivienda, el barrio y medio ambiente en la salud mental, y así generar estrategias de intervención específicas para nuestra realidad. Para ello, más que un enfoque basado en el individuo o la familia, se requiere una visión centrada en el sistema que establece y rige al individuo, a la familia, su ambiente y a la sociedad entera.

La vivienda sustentable. Con el fin de mostrar lo que corresponde a un proyecto de vivienda de interés social sustentable.

Lo que es cierto es que el acceso a la vivienda adecuada es un tema fundamental para lograr lo que Gaia ha denominado sustentabilidad integral (Gaia, 2005)², entendida como una categoría que implica tres dimensiones necesarias para lograr el desarrollo equitativo de los territorios: ambiental, económica y social. En el ámbito internacional el acceso a la vivienda adecuada forma parte de la agenda de la ONU y ha sido considerado entre los Objetivos de Desarrollo del Milenio (2000) y en el Programa UN-HÁBITAT (2002).

Desarrollo de la vivienda con aplicación de criterios de sustentabilidad. La construcción impacta fuertemente la vida de las personas y muy negativamente la salud del planeta. Se estima que los edificios utilizan el $50 \%$ de la energía disponible, consumen el $50 \%$ del agua y de los materiales sin tratar, el $80 \%$ de las tierras agrícolas que se pierden al año se destinan a la

1 Organización Panamericana de la Salud. Organización Mundial de la Salud; Colombia. Ministerio de la Protección Social; Colombia. Ministerio de Ambiente, Vivienda y Desarrollo Territorial. Bogotá, C0; 2003, Hacia una vivienda saludable: Que viva mi hogar: Manual para el facilitador.

2 GAJA DÍAZ, Fernando. Revolución Informacional, crisis ecológica y urbanismo. Guadalajara, Jalisco, México: Editorial Universidad de Guadalajara, 2005, 2da. Edición, p. 205. 
construcción y el $50 \%$ de la destrucción de los arrecifes es acusado de manera indirecta por la edificación ${ }^{3}$.

La vivienda de interés social en Colombia. Para autores como De Roux y Ramírez (2004), es un problema de tipo de derecho, es fundamental diferenciar los derechos según su naturaleza; los derechos civiles y políticos que exigen un compromiso del Gobierno y son de aplicación inmediata. Por su parte, los derechos económicos, sociales y culturales, como el caso de la vivienda, no son exigibles de inmediato como sí lo son los derechos civiles y políticos sino que son de exigibilidad gradual, "porque las obligaciones estatales correlativas son positiva o de acción, y están sometidas a la condición que las autoridades reúnan los recursos necesarios para darles cumplimiento". En este sentido, el cumplimiento implica políticas públicas graduales a fin de alcanzar paulatinamente la cobertura total de los DESC. En particular, se espera de un Estado una política de vivienda que cierre la brecha frente a la tenencia de vivienda y que proporcione vivienda digna, de calidad.

La política en este caso se orienta a facilitar la operación del mercado a través de estrategias que facilitan la oferta y otras que buscan hacer parte efectiva la demanda, en la primera clase se destacan la oferta competitiva en el mercado lograda mediante la eliminación de barreras a la entidad y a la optimización de la estructura de costos de las mismas. Por otro lado, es fundamental la eficiencia en el marco normativo de lo concerniente a la disposición de suelos y los trámites legales de vivienda tales como titulación, licencias de construcción, entre otros 4 .

En términos generales la política de vivienda de interés social en su mayoría está asociada a la eficiencia y acceso a la calidad de los servicios, en donde es evidente en el caso de la vivienda en Colombia, donde el Estado entrega competencias a los municipios, por ser estos los que cuentan con información más oportuna sobre las necesidades de la población y la manera en que potencialmente se pueden enfrentar a problemas asociados, en materia de vivienda de interés social, el marco legal tuvo sus inicios en la ley 9 de 1989 en la que se obligaba a todas las entidades territoriales a plantear planes de desarrollo, en los cuales deberían hacer en principio un plan de inversiones en distintos servicios como infraestructura pública y además obligaba a los municipios en el plan se especificara el área reservada para ejecutar planes de VIS, pero no existía como tal un conjunto de instituciones coordinadas para plantear y ejecutar la política en este tema.

En la actualidad, los recursos para el subsidio familiar de vivienda, son distribuidos principalmente a través de las cajas de compensación familiar (CCF), y a través del concurso de esfuerzo territorial. En la primera modalidad, los fondos son asignados a través de las CCF como representantes de FONVIVIENDA en algunas zonas del país. Tanto afiliados como no afiliados pueden postularse y la adjudicación final de los subsidios para VIS puede estar a cargo tanto de las CCF como de otras entidades. En la segunda modalidad, los recursos son asignados directamente por FONVIVIENDA, a través de la evaluación de los programas de soluciones de vivienda presentados por los municipios y los recursos son destinados a aquellos que demuestren una un proceso de planeación más estructurado 5 .

Voluntad política. Con respecto a la voluntad políti$\mathrm{Ca}$, se puede decir que la política de vivienda de interés social descentralizada no ha contado con un apoyo político continuo que movilice los intereses hacia su consolidación. Se observa que antes de 2003 el tema fue secundario en la agenda política. A partir del 2003, cuando se da inicio al fortalecimiento institucional en el sector y se homogeniza en el país la generación de los planes de ordenamiento territorial (POT), el tema da pasos para ubicarse en la agenda política. De ahí que la entrada tardía del tema de vivienda de interés social a la agenda política, frente a temas como educación y salud, explica en parte, el rezago en avances.

Sin embargo, es necesario reconocer que el diseño legal e institucional no ha sido suficiente para dar solución a esta problemática que en las dos últimas décadas se ha profundizado en los centros urbanos del país. Lo anterior se debe en parte a que en la planeación de la distribución de los recursos de la nación y de los entes territoriales para la inversión social la vivienda de interés social no es representativa respecto a los montos destinados para la prestación de servicios educación, salud, entre otros.

\section{MATERIAL Y MÉTODOS}

La ciudad de Barranquilla, capital del departamento del Atlántico, está ubicada en la zona noroccidental del departamento, a casi $1.000 \mathrm{~km}$ de distancia de

3 FONVIVIENDA. Programas y planeación de viviendas de interés Social, Bogotá, 2000.

4 POLÍTICAS DE VIVIENDA DE INTERÉS SOCIAL. Memorias del foro sobre políticas de vivienda de interés social, Manizales: Escuela Superior de Administración Pública, 1996.

5 FONVIVIENDA. Programas y planeación de viviendas de interés Social, Bogotá, 2000. 
Bogotá, la capital de Colombia. Gracias a su ubicación, la ciudad representa un nodo de desarrollo no sólo para todos los municipios del Atlántico, sino también para la gran mayoría de ciudades de la región Caribe colombiana.

El clima de Barranquilla es el tropical cálido seco y presenta anomalías y diferencias como consecuencia del sistema meteorológico local y de la ubicación geoastronómica.

La metodología utilizada es de tipo cualitativa ya que permite hacer la descripción de hechos reales y un análisis de los mismos. En tanto se intenta describir la nueva política habitacional, en comparación con la antigua política habitacional, a través del programa Fondo Solidario de Vivienda, es decir, inquirir sobre los elementos que la definen y caracterizan.

La investigación considera un uso combinado de estrategias de aproximación a la realidad que se quiere conocer, por un lado se realizara análisis temático a partir de textos sobre política habitacional y la segregación residencial,

El trabajo fue enmarcado dentro de un método ana-

lítico. Este método se caracteriza por ser más comprensivo, lo que le permitirá al investigador aplicar análisis generales de casos específicos, esto si se parte de que un problema es siempre cualitativo.

\section{RESULTADOS}

Está representada por la vivienda de interés social en el Distrito de Barranquilla.

Como primer paso se partió de la revisión de material bibliográfico, que permitió tener una mirada desde diferentes puntos de vistas y de enfoques. Seguidamente se centro esta investigación describir las características de la vivienda de interés social y el grado se sustentabilidad.

El enfoque utilizado es de tipo político - histórico y humanístico ya que permite tener una visión integral y multicausal de los hechos centrado en las políticas públicas de la vivienda de interés social, la distribución del espacio y las relaciones humanas.

En este artículo científico queda plasmada una de las recomendaciones realizadas por el investigador.

\section{MANUAL Y PROTOTIPO DE VIVIENDA INTERÉS SO- CIAL MÍNIMA SUSTENTABLE PARA BARRANQUILLA COLOMBIA}

Se hace evidente la creación de un proyecto alternativo que involucre el diseño, el urbanismo, la tecnolo- gía y materiales para mitigar problemas como vivienda y medio ambiente, si desconocer uno de los principales principios de la arquitectura el confort del ser humano, este trabajo no contempla si no la principal premisa ciclo de vida de los materiales y la arquitectura bioclimática como eje principal, "Las obras humanas, la urbanización, deben estar en el paisaje natural, no EN VEZ DE." Ricardo Barbetti

\section{DIMENSIONES MİNIMAS DEL LOTE}

La ubicación del lote debe ser aislada de los vecinos y su orientación más larga al eje eólico y la más corta hacia el sur.

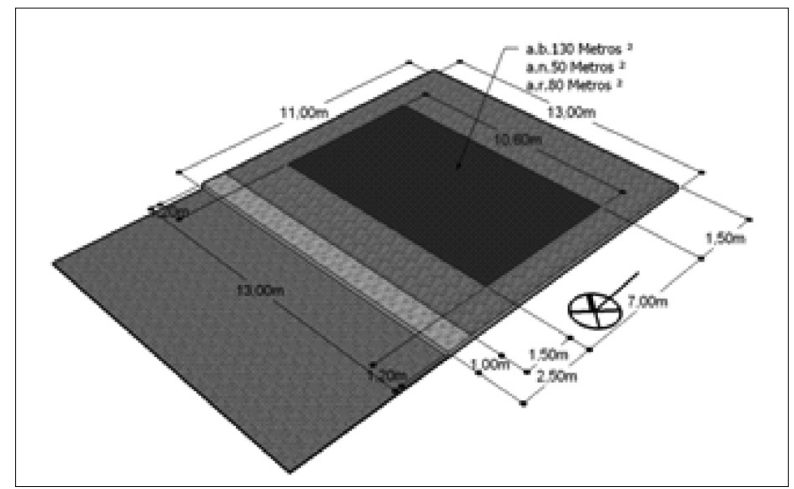

Figura 1. Lote mínimo.

Se deduce:

El ancho mínimo: $13.00 \mathrm{mts}$

El largo mínimo: $11 \mathrm{mts}$

Para un área bruta de lote de $130 \mathrm{mts} 2$

El agrupamiento por manzana tipo tablero de ajedrez (ver figura No.2) espaciado entre edificios. La distancia lateral entre edificios debe ser mínima una vez la altura de la vivienda y la distancia posterior mínimo una vez la altura.

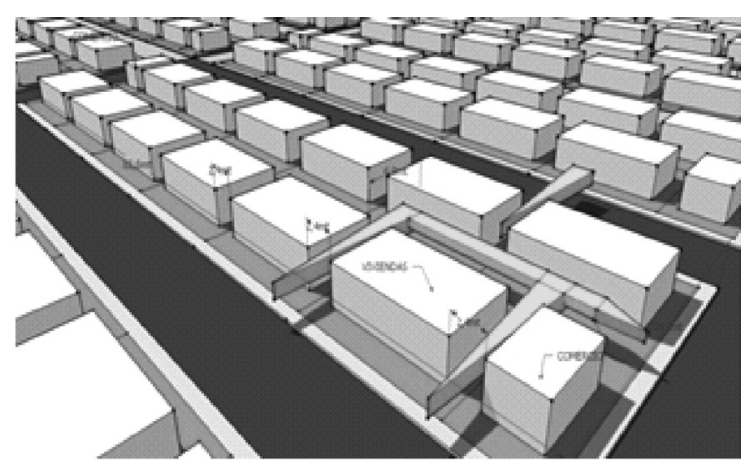

Figura 2. Loteo de manzana. 


\section{AISLAMIENTOS}

Aislamiento lateral: $1,2 \mathrm{mts}$

Aislamiento frontal: $2,0 \mathrm{mts}$

Aislamiento de fondo: $1,5 \mathrm{mts}$

Las condiciones para que una vivienda sea digna, no pueden partir solamente del hecho de tener un lugar de albergue. Estas deben estar ubicadas en espacios seguros con acceso a los servicios públicos básicos, en medio de comunidades productivas en las cuales se puedan desarrollar actividades comerciales y tener acceso a salud. (Ver figura No.3)

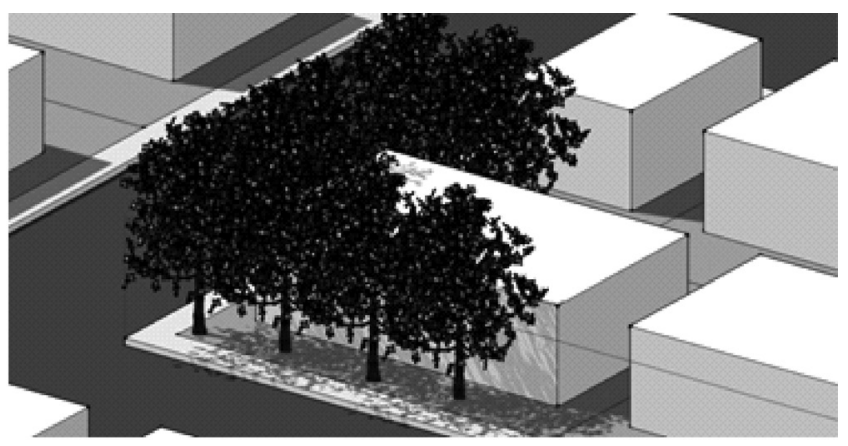

Figura 3. Diseño de antejardín con arborización nativa, adaptada al clima de la región, árboles frondosos y altos.

Jardín. El jardín es recomendable ya que sirve para dar un buen aspecto a la vivienda. Por otra parte la vegetación es el cemento que responde fielmente a las condiciones impuestas por los demás componentes del ecosistema; ya que eleva la cantidad de oxígeno, disminuye las partículas suspendidas y ruidos que se generen en la vivienda, así como también ayuda a eliminar radiación del calor que incidan directamente en el. (Ver figura No.4)

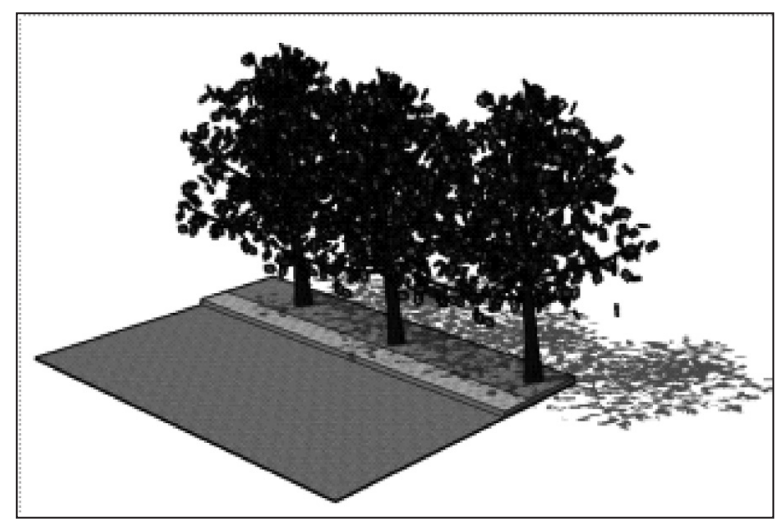

Figura 4. Jardín.
Como se sabe los árboles están adaptados muy bien el tipo de clima de la región y se presentan como árboles frondosos, altos y de hoja perenne. Por lo tanto en ellos se aplican conceptos como la de la ventilación que recorre por su inferior y es enfriada por la sombra. (Ver figura No.5).

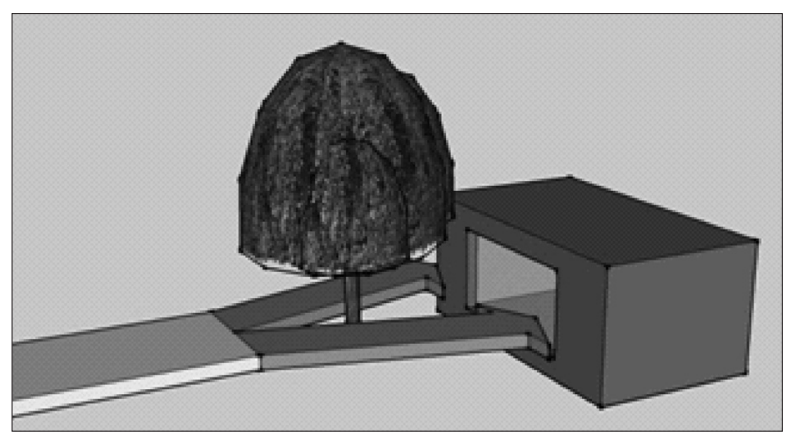

Figura 5. Esquema ventilación.

Sala. La sala es un espacio destinado a la convivencia familiar y social del habitante de esta ciudad, comúnmente estos espacios se sitúan en el área de recepción de la vivienda. (Ver figura No.6)
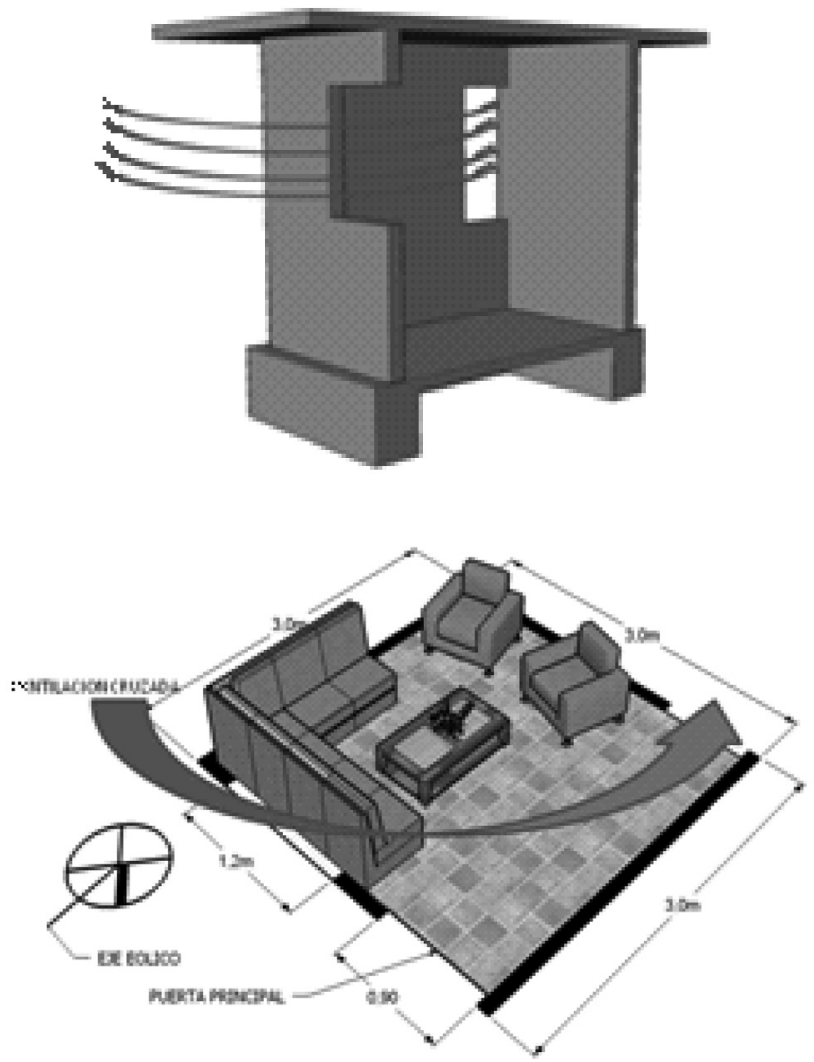

Figura 6. Esquema ventilación. 
Comedor. El comedor es un espacio destinado a la reunión familiar y es usado comúnmente para ingerir alimentos o comidas en reuniones especiales; Las medidas mínimas recomendables son de $3.00 \mathrm{mts} x$ $3.00 \mathrm{mts}$, y se orientara al eje eólico, con ventilación cruzada. (Ver figura No.7)

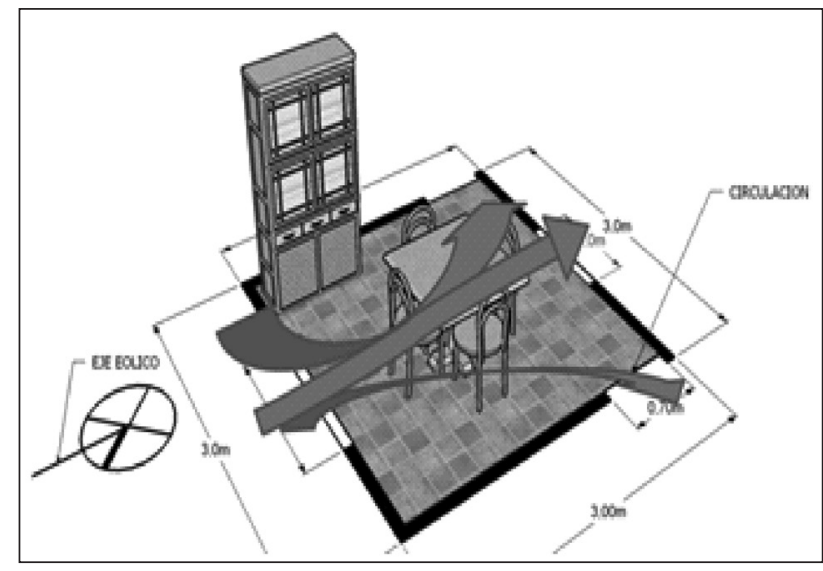

Figura 7. Diseño y estudio de área del comedor.

Habitaciones. Son sitios destinados al descanso de los ocupantes de la vivienda, estos sitios deben tener una excelente orientación, iluminación y ventilación, para un buen funcionamiento debe tener dimensiones mínimas de $2.70 \mathrm{mts} \times 2.80 \mathrm{mts}$, para condiciones del núcleo familiar de 5 a 6 personas promedio real de la ciudad. Ventilación de tipo cruzado y envolventes con aislantes térmicos para protección de asoleamiento excesivos. (Ver figura No.8 y No.9)

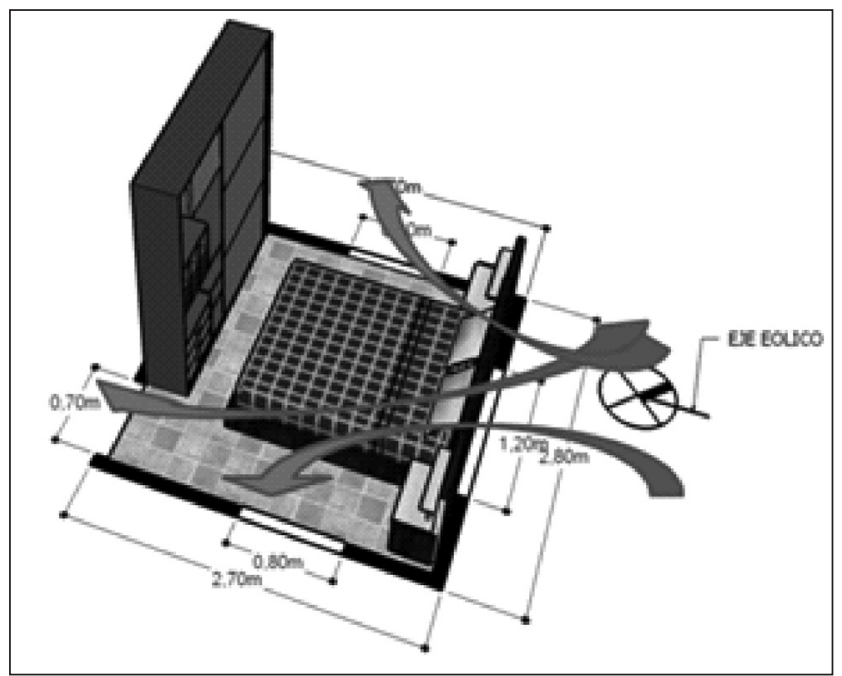

Figura 8. Diseño y estudio de áreas habitación tipo 1.

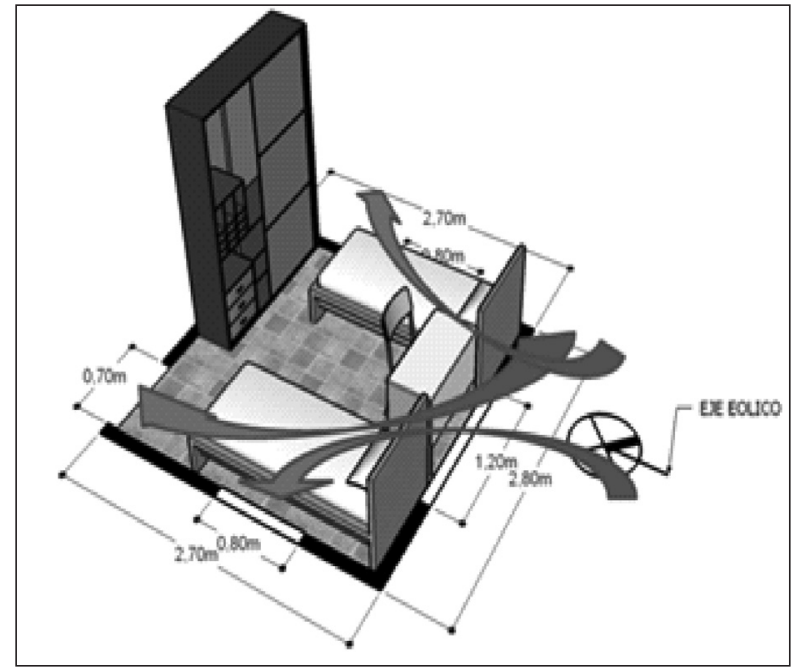

Figura 8. Diseño y estudio de áreas habitación tipo 2.

Baño. El baño generalmente es proyectado de dimensiones pequeñas; Se recomienda para este tipo de vivienda de $2.40 \mathrm{mts} \times 1.20 \mathrm{mts}$. Estos sitios son usados para el aseo personal y las necesidades fisiológicas que requiere el ser humano. (Ver figura No.10).

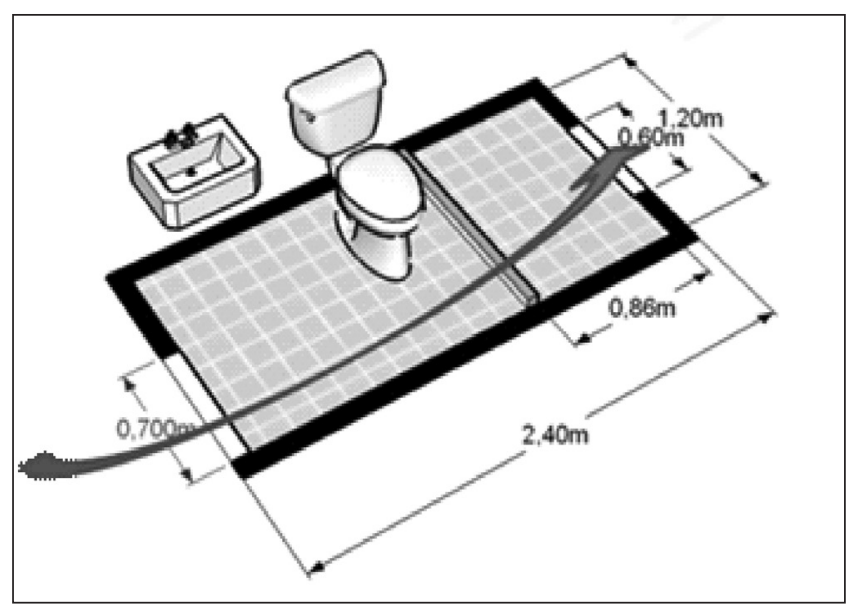

Figura 10. Diseño y estudio de áreas baño.

Patio. El patio es uno de los espacios que se requieren en la construcción de una vivienda ideal para una persona de esta ciudad. Las dimensiones de estos espacios por lo general deben ser proyectados a futuro, ya que en ellos se podrán idear ampliaciones a la vivienda, juegos para los niños, patios de servicios, etc. (ver figura No.11) 


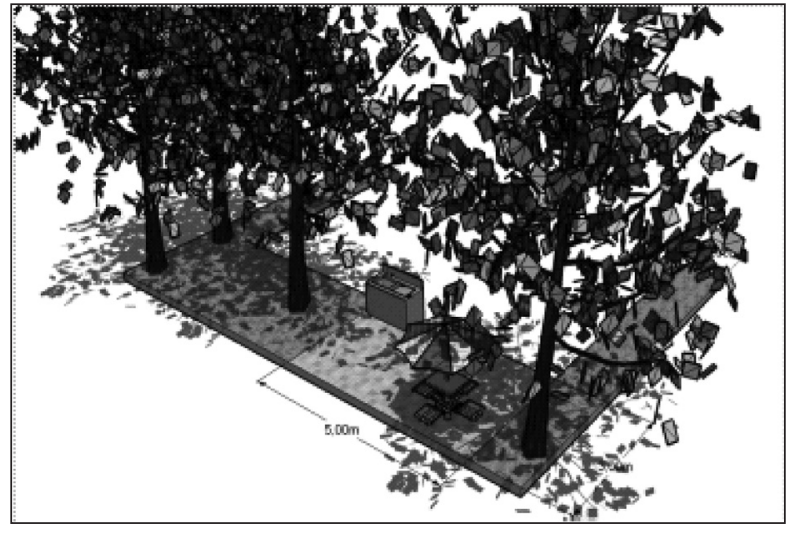

Figura 11. Al igual que el jardín el patio se diseña para uso de servicio, control climático y social.

Prefabricación. Para definir el proyecto de vivienda sustentable de bajo costo, se pensaría que la envolvente deberá ser un material que psicológicamente de seguridad de perduración, como el concreto el cual es sinónimo de fuerza y durabilidad.

Para esto y de acuerdo a las características del proyecto se remota el concepto y el sistema de prefabricación pesada de sistema cerrado, el cual presenta estas características: (Ver figura No.12)

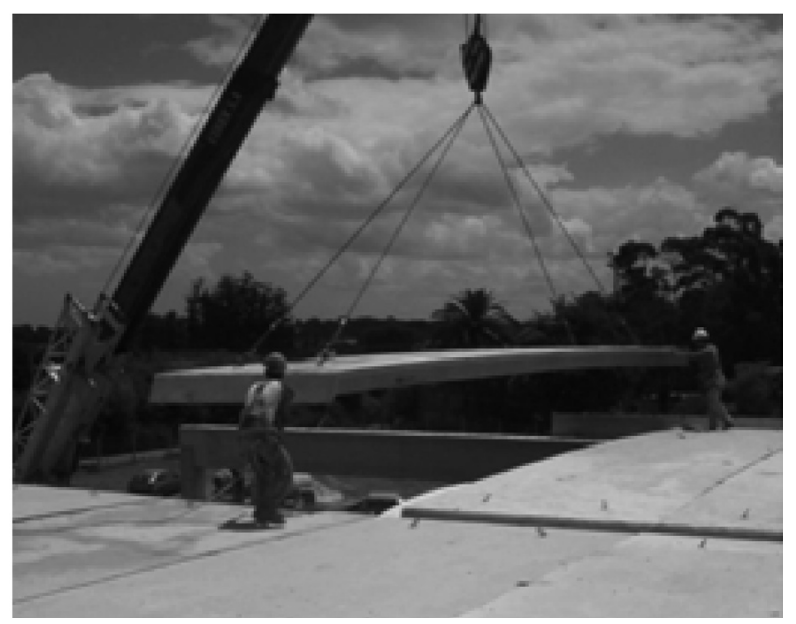

Figura 12. Transporte y montaje de elementos de prefabricación pesada.

1. Se producen en planta industrial todos sus componentes que luego se montan en obra.
2. Sistema de prefabricación total - resuelve totalmente la construcción del edificio, especialmente viviendas colectivas.

3. Disminución de cantidad de tareas y tiempos en obra.

4. Componentes de peso mayores a $500 \mathrm{~kg}$.

5. Sistema cerrado - se diseña, produce y monta todos los componentes coordinando con pocos subcontratos

6. Cimentación - combina con cualquier sistema de cimentación tradicional de los empleados en el medio.

Para dar solución a esta necesidad de vivienda, se buscó un método de producción masivo que permitiera afrontar cuantitativamente el problema, se pensó en un elemento tridimensional. "Que son aquellos cuyas partes que no están contenidas en el mismo plano de acuerdo con (Fernández 1974), clasificados según forma geométrica y el número de elementos que lo componen". Aunque estos elementos tridimensionales presentas grandes inconvenientes ya que según (Fernández 1974), se presenta como viviendas tipo "prisión" que limitan la libertad de la planta.

\section{PROTOTIPO}

\section{Materiales:}

1. Concreto aireado ${ }^{6}$ para muros, pisos y cubierta fundidos in situ (la utilización del cemento denominado "de carbono negativo" podría ser una alternativa muy viable en concordancia con la sustentabilidad).

2. Guadua: Encablescomosustituto delacerode refuerzo ${ }^{7}$ y corta soles de fachada.

3. Plástico reciclado para revestimientos de pisos, baños, cocina, tuberías de agua potable y sanitaria.

4. Caucho vulcanizado reciclado de llantas para ser utilizado como impermeabilizante y acabado exterior.

5. Madera: Puertas y ventanas

6. Vidrio libre de plomo: en ventanas

\section{Diseño}

Moderno - Racional - Funcional y utilización de la arquitectura bioclimática para lograr el confort.

\section{Modelo de Gestión}

Autogestión y Autoproducción

$6 \quad$ Características del concreto aireado: Aislante térmico y acústico por su bajo peso y densidad variable, Bombeable y autonivelante por su consistencia que varía de plástica a fluida.

$7 \quad$ Hidalgo, Oscar. Nuevas técnicas de construcción con bambú, Universidad nacional de Colombia, 1976. 


\section{PROPUESTA ESTRUCTURAL}

Consiste en un cubo rectangular con una malla en ambos sentidos de cables de guadua constituidos por 3 flejes de $2 \mathrm{~cm}$ de espesor entorchados entre sí. Creando una canasta alrededor de los vértices del cubo. Según estudios realizados por el Arq. Oscar Hidalgo, universidad Nacional de Colombia, sede Valle, demuestran que estos cables se asemejan a la resistencia de una varilla de acero de diámetro $1 / 2$ ".

Este proyecto tendrá los cálculos semejantes a un losa maciza con luz de diseño de $3.5 \mathrm{mt}$ con refuerzo de acero, peralte de $15 \mathrm{~cm}$. (ver figura 13)

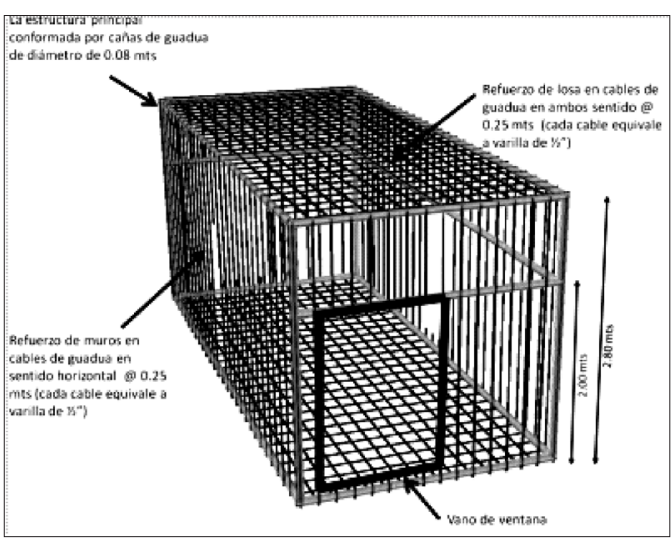

Figura 13. Representación gráfica de la prapuesta estructural.

\section{PROPUESTA ARQUITECTÓNICA BÁSICA}

Diseño constituido por dos módulos auto portantes construidos monolíticamente con un área de $49 \mathrm{mts} 2$, cuya altura interior no debe ser inferior a $2.70 \mathrm{mt}$.

Módulo 1: Dos alcobas y un baño. Área: 24, $5 \mathrm{mt} 2$ incluyendo muros y circulaciones.

Módulo 2: Sala, comedor, cocina y labores. Área: 24, $5 \mathrm{mts} 2$ incluyendo muros y circulaciones.

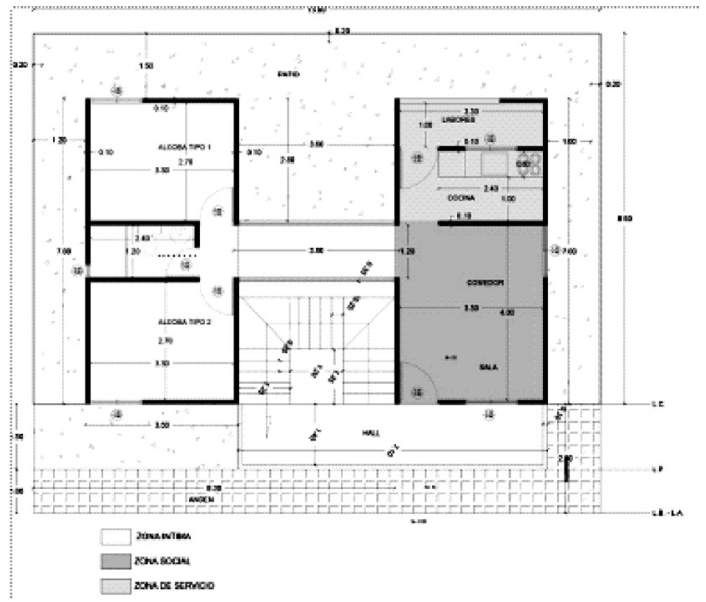

\section{PROPUESTA URBANA}

Reutilización del Suelo. Un paso importante en la concepción urbana moderna es la de la rehabilitación y el reusó de suelos en zonas deprimidas que hacen que la ciudad sea insegura. Esta propuesta va encaminada a la rehabilitación de alguna zona de la ciudad considerada "fea", para convertirla en foco de desarrollo. Otro de los dilemas de los planificadores actuales es expandir o densificar; la solución es densificar al reutilizar zonas dentro de la ciudad que por estar atomizadas dentro la urbe y al ser áreas pequeñas 0 medianas solo queda la altura.

"La principal premisa es anticiparse al aumento poblacional de acá a 40 años, contemplando pautas de dimensionamiento acordes a las demandas, partiendo del postulado de que las ciudades que se expanden horizontalmente degradan el suelo en forma irreversible, condicionando la implementación de infraestructuras de alto costo, con excesivo desplazamiento vehicular, y a partir de que el uso adecuado del espacio aéreo disponible genera ciudades de bajo impacto y bajo costo de mantenimiento, más seguras y con un estándar de vida más elevado."

Morfología Urbana Del Proyecto. En climas cálidos - secos, el trazado urbano tipo "damero" o "reticular", permite la maximización de la ventilación y la menor exposición directa del sol. Las manzanas tendrán la orientación de fachada más corta expuesta al sur y norte y las fachadas más largas expuestas hacia el este y oeste, con una ligera inclinación hacia el Nornoroeste para captar los vientos predominantes. (Ver figura 14)

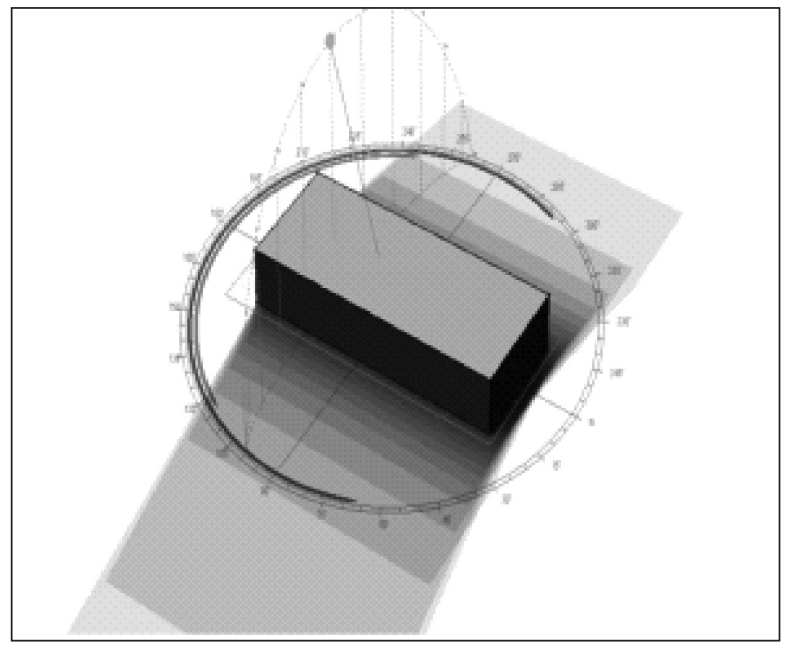

Figura 14. Representación gráfica solar y sombras. 
Sistema Vial. Las vías vehiculares de doble carril de $7 \mathrm{mt} 14$ con andén de $2.5 \mathrm{mt}$ arborizado, con vegetación nativa adaptada, de bajo consumo de agua, según el artículo "Índice de confort de la vegetación "15 del profesor de la Universidad del Atlántico, M.Sc. En Docencia Universitaria Orlando E. Jiménez González, el árbol que presenta la diferencia de temperatura más baja entre temperatura efectiva y temperatura de confort, es decir el que ofrece la sensación térmica más agradable, es el laurel (Ficus benjamina) con -1, $91^{\circ} \mathrm{C}$ por debajo de la temperatura de la ciudad.

Vías Peatonales. Andenes para peatones y área para vegetación y arborización. 2.5 mt. (ver figura 15)
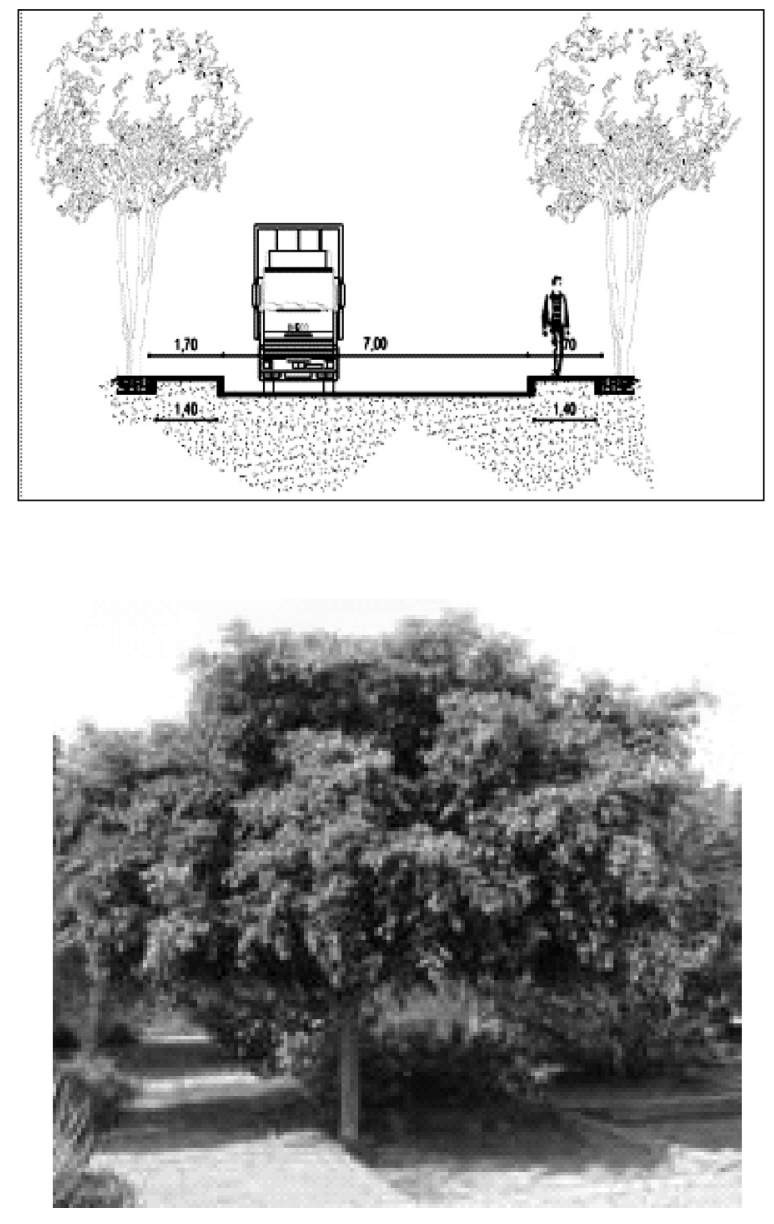

Figura 15. Laurel.
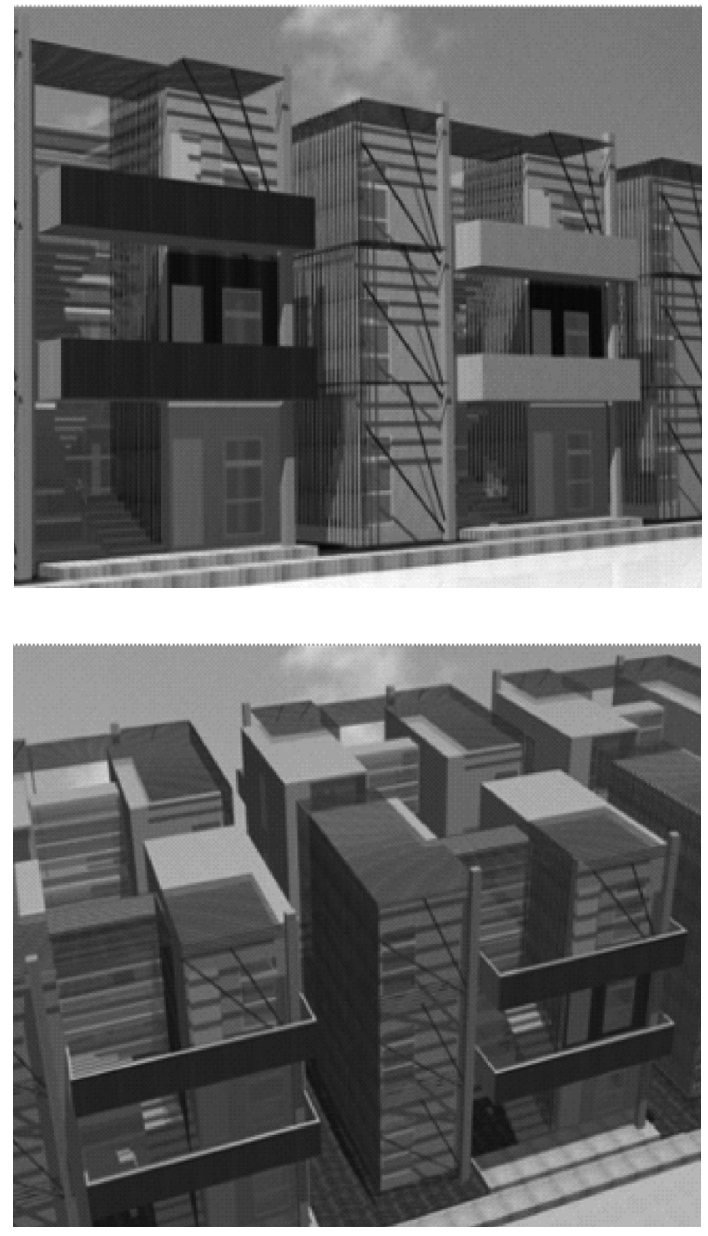

Figura 16. Representación gráfica de la propuesta arquitectónica.

\section{DISCUSIÓN}

La información tomada del censo 2005 para el déficit de vivienda en Barranquilla por localidades difiere en 14.363 viviendas con relación a las suministradas por el Ministerio de Medio Ambiente, Vivienda y Desarrollo Territorial en el documento plan estratégico para la habilitación de suelo para programas VIS. El déficit presentado incluyendo todos los municipios del área metropolitana fue de 36.151 viviendas con un déficit cuantitativo a 2005 de 24.671 viviendas, lo cual muestra una diferencia de 14.363 viviendas en comparación a la cifra 2005 de déficit de 39.034 arrojada por el DANE, de los cuales $87 \%$ son hogares urbanos con ingresos menores a 4 Salarios Mínimos Legales Mensuales (SMLM). ${ }^{8}$

8 Según el INFORME DE EVALUACION CALIDAD DE VIDA 2007-2008. Primer año de la administración de Alejandro Char. [documento en internet] disponible en: http://www.barranquillacomovamos.org/InformeBQCV2008DefinitivoFinal.pdf 
La Localidad del Sur Occidente es donde presenta mayor concentración el déficit cuantitativo de vivienda con el $62.2 \%$, la Localidad Sur Oriente en segundo lugar con el $29.8 \%$ y por último la Localidad Norte Centro Histórico presentó el $7.5 \%$ de déficit cuantitativo según el mismo Censo.

La Secretaría de Planeación Distrital señala que en el año 2005, la producción de vivienda para Barranquilla en el área urbana fue de 3.422 unidades, de éstas se construyeron 1.989 Viviendas de Interés Social (VIS) y 1.433 viviendas No VIS. Contrasta con el año 2006, donde las cifras muestran un decrecimiento del $63 \%$ en la producción de vivienda de interés social en área urbana.

Según los informes de CAMACOL, en el informe de la actividad edificadora en la ciudad de Barranquilla y su área metropolitana con corte al mes de agosto de 2009 los estratos 1 y 2 aportaron el $20 \%$ de la construcción y el estrato 3 participó con el $21 \%$ para un total de $41 \%$, siendo uno de los macroproyectos las 20 mil viviendas de interés social Villas de San Pablo, en la vía que comunica a Barranquilla con el corregimiento de
Una de las falencia de las políticas de vivienda de interés social radica en que se concibe la vivienda como una mercancía que ofrece el mercado de forma transparente pero para la cual la demanda no es efectiva en todos los casos, en este sentido la vivienda de acuerdo con las políticas y planes de desarrollo pierde todo el sustento social que las anima.

\section{REFERENCIAS}

FONVIVIENDA. Programas y planeación de viviendas de interés social, Bogotá, 2000. la brecha frente a la tenencia de vivienda

GAJA DÍAZ, Fernando. Revolución Informacional, crisis ecológica y urbanismo. Guadalajara, Jalisco, México: Editorial Universidad de Guadalajara, 2005, 2 da. Edición, p. 205.

Organización Panamericana de la Salud. Organización Mundial de la Salud; Colombia. Ministerio de la Protección Social; Colombia. Ministerio de Ambiente, Vivienda y Desarrollo Territorial. Bogotá, C0; 2003, Hacia una vivienda saludable: Que viva mi hogar: Manual para el facilitador.

POLÍTICAS DE VIVIENDA DE INTERÉS SOCIAL. Memorias del foro sobre políticas de vivienda de interés social, Manizales: Escuela Superior de Administración Pública, 1996. 